${ }^{1}$ Department of Dentistry, Universidade Federal do Rio Grande do Norte (UFRN), Natal, Rio Grande do Norte, Brazil.

${ }^{2}$ Department of Restorative Dentistry - School of Dentistry - Facult of Pharmacy, Dentistry and Nurse, Universidade Federal do Ceará (UFC), Fortaleza, Ceará, Brazil.
Corresponding author: Isauremi Vieira de Assunção 1787 Sen. Salgado Filho avenue, Natal - RN, 59056-000, Brazil; Mobile.: +55-84-99935-4263; Phone/Fax: +55-84-3215-4100; email: isauremi@gmail.com

Editor: Dr Altair A. Del Bel Cury

Received: July 3, 2021

Accepted: November 16, 2021

\section{Dual-Cured Adhesive System Improves Adhesive Properties of Dentin Cavities Restored with a Bulk-Fill Resin Composite}

\author{
Ana Margarida dos Santos Melo ${ }^{1}$ (iD, Anne Kaline \\ Claudino Ribeiro ${ }^{1}$ iD, Diana Araújo Cunha ${ }^{2}$ iD, Nara \\ Sousa Rodrigues ${ }^{2}$ iD, Vicente de Paulo Aragão \\ Saboia $^{2}$ (iD, Boniek Castillo Dutra Borges ${ }^{1}$ (iD), \\ Isauremi Vieira de Assunção ${ }^{1, *}$ iD
}

Aim: To evaluate the impact of a dual-cured adhesive system on the in situ degree of conversion (DC), bond strength (BS) and failure mode (FM) of adhesive interfaces in dentin cavities restored with a bulk-fill resin composite. Methods: 4-mm-deep dentin cavities with a 3.1 C-factor were created in 68 bovine incisors ( $n=17$ per group). The lightcured (Scotchbond ${ }^{\mathrm{TM}}$ Universal) or the dual-cured (Adper ${ }^{\mathrm{TM}}$ Scotchbond $^{\text {TM }}$ Multi-purpose Plus) adhesive system was applied to the cavities, which were then restored with a bulkfill resin composite (Filtek ${ }^{\text {TM }}$ Bulk Fill). In situ DC analysis was performed by means of micro Raman spectroscopy at the top and bottom interfaces. Push-out BS was measured in a universal testing machine after 24-h or 6-month water storage. FM was determined with a stereomicroscope. Data of in situ DC and BS were analyzed by two-way analysis of variance (ANOVA) and Tukey test $(p<0.05)$, while the FM was analyzed descriptively. Results: The groups that received the dual-cured adhesive system showed statistically higher in situ DC and BS than those that received the light-cured adhesive system. Cohesive failure mode was the most frequent in all conditions. Conclusion: In situ DC and BS were influenced by the curing strategies of the adhesive systems with better performance of the dual-cured material.

Keywords: Aging. Composite resins. Polymerization. Dentinbonding agents. Physical Properties. 


\section{Introduction}

The proper cure of the adhesive system is one of the factors needed to achieve the required mechanical properties ${ }^{1}$ and longevity ${ }^{2,3}$ of dental restorations. Inappropriate monomer conversion and the consequent residual monomers in the hybrid layer increase the local permeability and water sorption ${ }^{2-4}$, weakening the hybrid layer structure, and impairing the adhesive capacity 4 . Therefore, a higher degree of conversion of the adhesive system, would provide longer-lasting adhesive integrity to dental restorations.

The dual-curing strategy can promote the material self-cure when a proper light activation might be compromised. Therefore, its use is traditionally recommended in indirect restorations or fiber post bonding in the root canal ${ }^{5}$. The benzoyl peroxide and tertiary amine in the catalyst allow the cure reaction without light energy ${ }^{6}$, which can increase the degree of conversion and, consequently, promote long-lasting bonds.

Areas of the cavity preparation for direct resin composite restorations that are too far from the curing light tip may receive insufficient energy, negatively affecting the degree of conversion and bond stability ${ }^{7}$. To overcome this inconvenience, the use of dual-cured adhesive systems has been suggested in 2-mm-deep cavities resulting in greater dentin bonding durability ${ }^{8}$, probably due to their higher degree of conversion in the hybrid layer and faster cure. However, when such tooth preparations occur in posterior teeth, the tip of the curing light can be set even further than $2 \mathrm{~mm}$ in certain dentin sites, negatively affecting interfacial properties in such areas. Thus, regular viscosity bulk-fill resin composites, which allow light-curing single increments of up to 4 to $5 \mathrm{~mm}$ thickness ${ }^{9}$, could be used to fill deep preparations with a single resin composite increment, saving chair time. However, it is not well known if using a dual-cured adhesive system in 4-mm-depth would benefit the adhesive conversion and dentin bonding durability.

Thereby, the aim of this study was to evaluate the in situ degree of conversion (DC) of light-cured and dual-cured adhesive systems and their impact on bond strength (BS) and failure modes (FM) in 4-mm-deep dentin cavities restored with a regular viscosity bulk-fill resin composite. The null hypothesis tested was that different curing strategies of adhesive systems and storage times would not affect the physical properties of the adhesive interface.

\section{Materials and Methods}

\section{Experimental Design and Specimen Preparation}

This in vitro study involved the following response variables: in situ DC, BS and FM. For in situ DC $(n=17)$, the factors studied were two adhesive systems with different curing strategies (light-cured: Scotchbond ${ }^{\text {TM }}$ Universal, 3M ESPE, St. Paul, USA; and dual-cured: Adper ${ }^{\mathrm{TM}}$ Scotchbond ${ }^{\mathrm{TM}}$ Multi-purpose Plus, 3M ESPE, St. Paul, USA), and the regions of the specimen (top and bottom). For BS and FM ( $n=17)$, the factors studied were the two adhesive systems with different curing strategies and the time of water storage ( $24 \mathrm{~h}$ and 6 months aging). Materials used in this study are described in Table 1. 
Table 1. Materials used in this study.

\begin{tabular}{|c|c|c|}
\hline Material & Chemical composition (wt\%) & Lot number \\
\hline $\begin{array}{l}\text { Filtek }^{\text {TM }} \text { Bulk Fill (3M } \\
\text { ESPE, St. Paul, MN, USA) }\end{array}$ & $\begin{array}{l}\text { Silane treated ceramic }(60-70) \\
\text { Aromatic urethane dimethacrylate }(10-20) \\
\text { Diurethane dimethacrylate }(\text { UDMA })(1-10) \\
\text { Ytterbium fluoride }(\text { YbF3) }(1-10) \\
\text { Silane treated silica }(1-10) \\
\text { 1,12-dodecane dimethacrylate (DDDMA) }(<10) \\
\text { Silane treated zirconia }(<5) \\
\text { Water }(<5)\end{array}$ & 1717800606 \\
\hline $\begin{array}{l}\text { Scotchbond } d^{\mathrm{TM}} \text { Universal } \\
\text { ( } 3 \mathrm{M} \text { ESPE, St. Paul, } \\
\text { MN, USA) }\end{array}$ & $\begin{array}{l}\text { 2-hydroxyethyl methacrylate }(15-25) \\
\text { Bisphenol A diglycidyl ether dimethacrylate (BisGMA) }(15-25) \\
\text { 2-propenoic acid, 2-methyl-, reaction products with } \\
\text { 1,10-decanediol and phosphorous oxide (P2O5) }(10-20) \\
\text { Ethanol }(10-15) \\
\text { Water }(10-15) \\
\text { 2-propenoic acid, 2-methyl-, 3-(trimethoxysilyl)propyl ester, } \\
\text { reaction products with vitreous sílica }(7-13) \\
\text { Copolymer of acrylic and itaconic acid }(1-5) \\
\text { Camphorquinone }(<2) \\
\text { Dimethylaminobenzoat }(-4)(<2) \\
\text { (Dimethylamino)ethyl methacrylate }(<1)\end{array}$ & 1718500452 \\
\hline \multirow{3}{*}{$\begin{array}{l}\text { Adper }^{T M} \text { Scotchbond } \\
\text { Multi-purpose Plus (3M } \\
\text { ESPE, St. Paul, MN, USA) }\end{array}$} & $\begin{array}{l}\text { Primer: } \\
\text { Water }(40-50) \\
\text { 2-hydroxyethyl methacrylate (HEMA) }(35-45) \\
\text { Copolymer of acrylic and itaconic acids }(10-20)\end{array}$ & 1723700298 \\
\hline & $\begin{array}{l}\text { Activator: } \\
\text { Ethyl alcohol }(>95) \\
\text { Sodium benzenesulfinate }(<5)\end{array}$ & 1728600477 \\
\hline & $\begin{array}{l}\text { Catalyst: } \\
\text { Bisphenol A diglycidyl ether dimethacrylate (BisGMA) }(60-70) \\
\text { 2-hydroxyethyl methacrylate (HEMA) }(30-40) \\
\text { Benzoyl peroxide }(<2.5) \\
\text { Triphenylantimony }(<0.5) \\
\text { Triphenylphosphine }(<0.5) \\
\text { Hydroquinone }(<0.05)\end{array}$ & 1727600198 \\
\hline
\end{tabular}

Source: Safety Data Sheet (SDS)

Figure 1 shows a schematic representation of the specimens' preparation and analyses performed. Sixty-eight bovine incisors without enamel cracks or structural defects were selected for dental preparations according to a previously described method ${ }^{10,11}$. Teeth were decontaminated in a water solution of thymol $(0.1 \%)$ at $4^{\circ} \mathrm{C}$ for a week, and then the roots were removed at the cementoenamel junction (CEJ) with a diamond saw using a precision cutting machine (Isomet 1000; Buehler, Lake Forest, IL, USA) under irrigation. Such surface will be the future top of the sample. A parallel cut was made $4 \mathrm{~mm}$ from the CEJ, surface that will be the bottom of the sample, resulting in a 4-mm-high specimen. The top and bottom surfaces of the specimens were sanded with 400 and 600 grits sandpapers (Labopol-21, Struers, Copenhagen, Denmark). The central void of the specimen was prepared with Maxicut burs (Komet Inc, Lemgo, Germany) mounted in a handpiece under air-water cooling, resulting in standardized conical cavities $(4.8 \times 2.8 \times 4 \mathrm{~mm})$ with a $3.1 \mathrm{C}$-factor. 


\section{A}
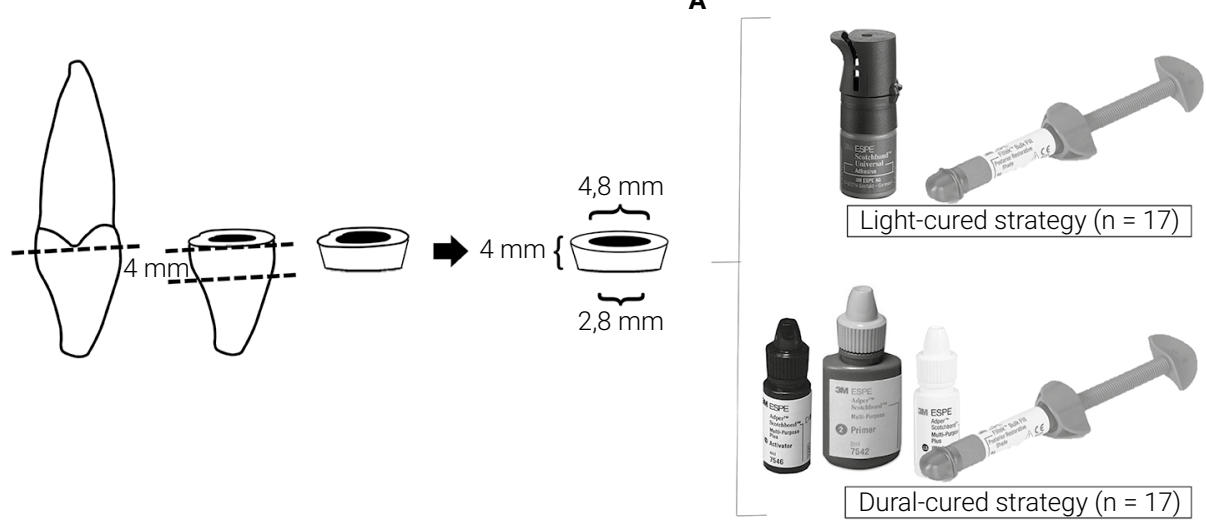

B


Figure 1. Specimen preparation and groups of curing strategies (A). Regions of specimens (B). Storage times (C). Analysis of the in situ degree of conversion through the micro-Raman spectroscopy (D). Analysis of the bond strength in the universal testing machine (UTM) (E). Analysis of failure mode through Scanning Electron Microscopy (SEM) (F).

Curing strategies were as follow: Light-cured: No acid etching was done. The adhesive was applied and rubbed for $20 \mathrm{~s}$, followed by gentle air drying for approximately $5 \mathrm{~s}$ to evaporate the solvent, and light cured for $10 \mathrm{~s}$ (Coltolux LED, Coltène/Whaledent, Altstätten, Switzerland - $1200 \mathrm{~mW} / \mathrm{cm}^{2}$ ). Dual-cured: The surface was etched with $37 \%$ phosphoric acid for $15 \mathrm{~s}$ and rinsed for $30 \mathrm{~s}$. Dentin was maintained moist after water excess removal with absorbent paper. The activator was applied and air-dried gently for $5 \mathrm{~s}$. Then, the primer was applied and air dried gently for $5 \mathrm{~s}$, leaving the surface with a shiny aspect. A coat of the catalyst was applied and light-cured for $10 \mathrm{~s}$ 
(Coltolux LED - $1200 \mathrm{~mW} / \mathrm{cm}^{2}$ ). The adhesive system was applied according to the manufacturers' instructions. The bond component was not applied because it is part of the Adper Scotchbond Multi-purpose Plus catalyst formulation.

After adhesive procedures, the teeth were set on a glass slab and filled with a single increment of Filtek ${ }^{\text {TM }}$ Bulk Fill (3M ESPE, St. Paul, MN, USA) resin composite. A glass slide was pressed onto the specimen in order to standardize the smooth surface and the distance of $1,0 \mathrm{~mm}$ between the light source and the resin composite during photoactivation. The resin composite was cured for $20 \mathrm{~s}$ (Coltolux LED - $1200 \mathrm{~mW} / \mathrm{cm}^{2}$ ). All the adhesive, restorative and curing procedures were performed by a single operator. Samples were polished with 400, 500, and 800 grit sandpapers and cleaned in an ultrasonic bath for $20 \mathrm{~min}$. Half of samples were stored in distilled water at $37^{\circ} \mathrm{C}$ for 24 hours. Another thirty four samples were stored at the same conditions for aging in distilled water changed weekly for 6 months.

\section{"In Situ” Degree of Conversion (DC)}

The degree of conversion (DC) of each material tested in this study was evaluated through micro-Raman spectroscopy (Xplora micro-Raman, Horiba, Paris, France). Spectroscope calibration was done using a Silicon sample. Raman spectra were collected using in the range between 1590 and $1670 \mathrm{~cm}^{-1}$ using the 638 $\mathrm{nm}$ laser emission wavelength with $10 \mathrm{~s}$ acquisition time and 3 accumulations. The diameter of the laser beam used over the specimen was $1 \mu \mathrm{m}$ and the analysis was performed with a 100x magnification lens (Olympus UK, London, UK). The spectrum was obtained in the middle of hybrid layer. Six random spots of each sample were analyzed (three at the top and three at the bottom). A noncured amount of each adhesive system was used as reference. Processing was performed with the Opus Spectroscopy Software version 6.5 (Bruker Optik GmbH, Ettlingen, Baden-Wurttemberg, Germany).

The $\%$ DC was calculated using the monomers to polymer double bonds ratio in the adhesive, according the formula (I) below, in which " $\mathrm{R}$ " is the ratio between aliphatic and aromatic bond peaks at 1635 and $1605 \mathrm{~cm}^{-1}$ in the adhesive layer, as performed in a previous study ${ }^{12}$.

$\mathrm{DC}(\%)=100 \times\left(1-\left[\mathrm{R}_{\text {cured }} / \mathrm{R}_{\text {uncured }}\right]\right)$

\section{Bond Strength (BS) Evaluation}

BS was evaluated by the push-out method in a universal testing machine (EMIC DL 2000, São José dos Pinhais, PR, Brazil) as previously reported ${ }^{10,11}$. An acrylic device with a central hole was adapted on the machine base where specimens were placed with its larger surface facing down. The smaller surface was pushed by a cylindrical plunger (1-mm diameter) with a compressive force $(0.5 \mathrm{~mm} / \mathrm{min})$ until failure. Data were obtained in $\mathrm{N}$ and transformed in MPa using the following equation (II), in which " $N$ " is the bond strength in Newton, " $\mathrm{R}$ " is the larger surface radius, and " $r$ " is the smaller surface radius and " $\mathrm{h}$ " is the sample height ${ }^{10,11}$.

$\mathrm{MPa}=\mathrm{N} / \pi(R+r)\left[\left(h^{2}+(R-r)^{2}\right]\right.$ 


\section{Failure Mode (FM) Analysis}

After BS test, the fractured specimens were examined on the surface of the dentin and on surface of resin composite using a stereomicroscope (Stereo Zoom, Bausch \& Lomb, New York, NY, USA) at 30x magnification. The failure modes were categorized into cohesive (dentin or resin composite failure), adhesive (dentin/resin composite interface failure), or mixed (cohesive failure in resin composite and adhesive in interface), as previously described ${ }^{13}$.

\section{Statistical Analysis}

Data normality and homoscedasticity were analyzed through, respectively, D'Agostino \& Pearson test $(p>0.05)$ and Bartlett's test in GraphPad Prism 7 software (San Diego, CA, USA). In situ DC and BS data were analyzed using two-way ANOVA and Tukey's post hoc tests $(p<0.05)$ in GraphPad Prism 7 software. Failure modes patterns were descriptively analyzed.

\section{Results}

\section{"In Situ" Degree of Conversion}

Significant differences in DC were found between curing strategies $(p<0.01)$. Multiple comparisons are shown in Table 2. Top and bottom interfaces showed similar in situ DC, while the dual-cured adhesive system showed higher DC than light-cured adhesive system in both regions.

Table 2. Means (standard deviations) of in situ DC (\%) according to the curing strategy of the adhesive system and specimen region.

\begin{tabular}{lll}
\hline \multirow{2}{*}{ Region } & \multicolumn{2}{c}{ Curing strategy } \\
\cline { 2 - 3 } & Light-cured & Dual-cured \\
\hline Top & $53.4(8.6)^{\mathrm{Ba}}$ & $62.6(6.9)^{\mathrm{Aa}}$ \\
\hline Bottom & $54.4(8.9)^{\mathrm{Ba}}$ & $62.2(10.1)^{\mathrm{Aa}}$ \\
\hline
\end{tabular}

Distinct uppercase letters indicate statistically significant differences between curing strategies for the same region $(p<0.05)$. Distinct lowercase letters indicate statistically significant differences between regions for the curing strategy $(p<0.05)$.

\section{Bond Strength}

Significant differences were found between curing strategies $(p<0.05)$, storage times $(p<0.01)$, and the interaction between the two factors was significant $(p<0.01)$. Multiple comparisons are shown in Table 3. Dual-cured adhesive system provided higher BS then light-cured adhesive system in both storage times. For light-cured material, 24-h and 6-month storage times provided similar BS. Specimens bonded with dualcured adhesive system stored for 6 months provided lower BS than those stored for $24 \mathrm{~h}$, even though they showed higher BS than the light-cured specimens. 
Table 3. Means (standard deviations) of BS (MPa) according to the curing strategy of the adhesive system and storage time in water.

\begin{tabular}{lcc}
\hline \multirow{2}{*}{ Storage time } & \multicolumn{2}{c}{ Curing strategy } \\
\cline { 2 - 3 } & Light-cured & Dual-cured \\
\hline $24 \mathrm{~h}$ & $7.7(1.4)^{\mathrm{Ba}}$ & $12.2(1.5)^{\mathrm{Aa}}$ \\
\hline 6 months & $8.0(0.7)^{\mathrm{Ba}}$ & $9.7(0.7)^{\mathrm{Ab}}$ \\
\hline
\end{tabular}

Distinct uppercase letters indicate statistically significant differences between curing strategies for the same storage time $(p<0.05)$. Distinct lowercase letters indicate statistically significant differences between storage times for the curing strategy $(p<0.05)$.

\section{Failure mode}

Most failures were of cohesive mode regardless of the adhesive and storage time. The light-cured adhesive system showed more adhesive failures than the dual-cured (Table 4).

Table 4. Number of adhesive, cohesive, and mixed failures according to curing strategy of adhesive systems and storage times.

\begin{tabular}{lcccccc}
\hline \multicolumn{7}{c}{ Curing strategy } \\
\hline Storage time & Adhesive & Cohesive & Mixed & Adhesive & Cohesive & Mixed \\
\hline $24 \mathrm{~h}$ & 6 & 8 & 3 & - & 13 & 4 \\
\hline 6 months & 3 & 13 & 1 & 2 & 9 & 6 \\
\hline Total & 9 & 21 & 4 & 2 & 22 & 10 \\
\hline
\end{tabular}

\section{Discussion/Conclusion}

The null hypothesis tested - that different curing strategies of adhesive systems and storage times do not affect physical properties of adhesive interfaces - was rejected, as specimens in which dual-cured adhesive system was used had significant differences in the analyzed variables.

An universal adhesive system was chosen as the light-cured material since it represents the newest eighth-generation adhesives that can be applied according to the dentist's preference, with or without dentin etching (etch-and-rinse or self-etch application $)^{14}$. Previous studies showed that Scotchbond ${ }^{\mathrm{TM}}$ Universal adhesive presents similar dentin bond strength ${ }^{15}$ and bond stability ${ }^{16}$ for etch-and-rinse and self-etch techniques, or higher bond strength for self-etch technique ${ }^{17}$, which was used in this investigation.

Although in situ DC was similar for top and bottom regions with both curing strategies, the dual-cured adhesive system provided higher DC and BS than the light-cured. The dual-cured adhesive used in this study requires the application of an activator and a catalyst before and after the primer, respectively. The activator contains components derived from sulfinate salts, such as Sodium benzenesulfinate, that react with acidic monomers of the primer to produce phenyl or benzenesulfonyl free radicals and initiate polymerization ${ }^{18}$. The catalyst contains benzoyl peroxide that can chemically acti- 
vate the polymerization reaction without light energy by reacting with tertiary amine to produce free radicals ${ }^{6}$. These characteristics can contribute to increase the degree of conversion of the adhesive system in order to promote long-lasting bonds, which could justify the better results obtained with the dual-cured adhesive system to in situ $\mathrm{DC}$ and $\mathrm{BS}$.

In addition, higher DC values were reported for multi-step adhesives compared to simplified adhesive systems due to the relatively hydrophobic and un-solvated bond layer from the primer of multi-step adhesive systems ${ }^{3,19}$. On the other hand, the higher percentage of hydrophilic monomers and water presence in simplified adhesives (as Scotchbond ${ }^{\mathrm{TM}}$ Universal) impair the curing reaction ${ }^{20}$, decreasing DC.

Although differences regarding bond strength were found among groups, the low occurrence of adhesive failures may reflect an adequate interaction between materials and dentin ${ }^{21}$. The decreased BS values after 6-month aging for dual-cured strategy is possibly associated with the degradation of collagen fibers exposed to acid etching that were not completely covered by the primer, enabling the enzymatic action on matrix metalloproteinases (MMPs) and cysteine cathepsins ${ }^{22}$. On the other hand, as the light-cured strategy had a universal adhesive system applied in self-etching mode, it is likely that fewer collagen fibrils were left uncovered after adhesive application ${ }^{23}$, favoring bond stability. Although presenting lower BS values after water storage, the dual-cured adhesive system provided higher BS means than the light-cured material, which is an important finding. This might have been due to the better mechanical strength of multi-step adhesive systems, which is in part related to their higher DC. Further studies should be conducted to evaluate bonding stability of dual-cured adhesives after longer aging times.

The results of this laboratorial investigation indicated that the use of a dual-cured adhesive system may result in higher DC within the hybrid layer and better bonding performance, increasing the longevity of tooth restorations.

The choice for a three-step dual-cured adhesive system was made since three-step adhesive systems are the gold standard ${ }^{24}$. However, there are chemical activators that can transform some universal adhesives into dual-cured adhesives. Therefore, the stability of dentin bond strength after 6-month water storage has been previously reported for Scotchbond ${ }^{\mathrm{TM}}$ Universal with the self-etching technique ${ }^{16}$. Thus, further investigations should be performed to compare which dual-cured material would provide better performance, such as the outcomes of dual-cured in cavities filled by the incremental technique and longer aging times.

Concerning the findings of this study, the dual-cured adhesive system influenced the bond strength and degree of conversion in 4-mm deep dentin cavities filled with a bulk-fill resin composite. Although the dual-cured adhesive bond strength had reduced after 6 months, it was higher compared to the light-cured independently of the storage time. The degree of conversion was greater in both regions for the dualcured adhesive system. These results suggest a positive effect on bond strength, and better performance of the dual-cured adhesive system, which might contribute to the success and longevity of resin composite restoration in deep cavities filled with a bulk-fill resin composite. 


\section{Data availability}

Datasets related to this article will be available upon request to the corresponding author.

\section{References}

1. Ferracane $\mathrm{JL}$, Greener $\mathrm{EH}$. The effect of resin formulation on the degree of conversion and mechanical properties of dental restorative resins. J Biomed Mater Res. 1986 Jan;20(1):121-31. doi:10.1002/jbm.820200111.

2. Breschi L, Mazzoni A, Ruggeri A, Cadenaro M, Di Lenarda R, De Stefano Dorigo E. Dental adhesion review: aging and stability of the bonded interface. Dent Mater. 2008 Jan;24(1):90-101. doi: 10.1016/j.dental.2007.02.009.

3. Cadenaro M, Antoniolli F, Sauro S, Tay FR, Di Lenarda R, Prati C, et al. Degree of conversion and permeability of dental adhesives. Eur J Oral Sci. 2005 Dec;113(6):525-30. doi: 10.1111/j.1600-0722.2005.00251.x.

4. Tay FR, Pashley DH, Suh BI, Carvalho RM, Itthagarun A. Single-step adhesives are permeable membranes. J Dent. 2002 Sep-Nov;30(7-8):371-82. doi: 10.1016/s0300-5712(02)00064-7.

5. Faria-e-Silva AL, Casselli DS, Lima GS, Ogliari FA, Piva E, Martins LR. Kinetics of conversion of two dual-cured adhesive systems. J Endod. 2008 Sep;34(9):1115-8. doi: 10.1016/j.joen.2008.06.016.

6. Arrais CA, Rueggeberg FA, Waller JL, de Goes MF, Giannini M. Effect of curing mode on the polymerization characteristics of dual-cured resin cement systems. J Dent. 2008 Jun;36(6):418-26. doi: 10.1016/j.jdent.2008.02.014.

7. Shirai K, De Munck J, Yoshida Y, Inoue S, Lambrechts P, Suzuki K, et al. Effect of cavity configuration and aging on the bonding effectiveness of six adhesives to dentin. Dent Mater. 2005 Feb;21(2):110-24. doi: 10.1016/j.dental.2004.01.003.

8. Borges BC, Vilela AR, da Silva-Junior CA, Souza-Junior EJ, Sinhoreti MA, Pinheiro FH, et al. Dual-cured etch-and-rinse adhesive systems increase the bond durability of direct coronal dentin restorations. Oper Dent. 2013 Sep-Oct;38(5):512-8. doi: 10.2341/12-246-L.

9. Campodonico CE, Tantbirojn D, Olin PS, Versluis A. Cuspal deflection and depth of cure in resin-based composite restorations filled by using bulk, incremental and transtooth-illumination techniques. J Am Dent Assoc. 2011 Oct;142(10):1176-82. doi: 10.14219/jada.archive.2011.0087.

10. Sousa-Lima RX, Silva L, Chaves L, Geraldeli S, Alonso R, Borges B. Extensive assessment of the physical, mechanical and adhesion behavior of a low-viscosity bulk fill composite and a traditional resin composite in tooth cavities. Oper Dent. 2017 Sep/Oct;42(5):E159-66. doi: 10.2341/16-299-L.

11. Chaves LVF, Oliveira SN, Özcan M, Acchar W, Caldas MRGR, Assunção IV, et al. Interfacial properties and bottom/top hardness ratio produced by bulk fill composites in dentin Cavities. Braz Dent J. 2019 Oct 7;30(5):476-83. doi: 10.1590/0103-6440201902741.

12. Rodrigues NS, Souza LC, Feitosa VP, Loguercio AD, D'Arcangelo C, Sauro S, et al. Effect of different conditioning/deproteinization protocols on the bond strength and degree of conversion of self-adhesive resin cements applied to dentin. Int J Adhes Adhes. 2018 Mar;81:98-104. doi: 10.1016/j.ijadhadh.2017.03.013.

13. Sousa-Lima RX, Melo AMS, Guimarães LKC, Souza ROA, Caldas MRGR, Assunção IV, et al. Mechanical properties of low and regular viscosity bulk fill composites in a 3D dentin cavity model. J Adhes Sci Tech. 2020 Sep 10; 35(3):325-35. doi: 10.1080/01694243.2020.1802134.

14. Van Meerbeek B, Yoshihara K, Van Landuyt K, Yoshida Y, Peumans M. From Buonocore's pioneering acid-etch technique to self-adhering restoratives. A status perspective of rapidly advancing dental adhesive technology. J Adhes Dent. 2020 22(1):7-34. doi: 10.3290/j.jad.a43994. 
15. Chen C, Niu LN, Xie H, Zhang ZY, Zhou LQ, Jiao K, et al. Bonding of universal adhesives to dentine Old wine in new bottles? J Dent. 2015 May;43(5):525-36. doi: 10.1016/j.jdent.2015.03.004.

16. Muñoz MA, Luque-Martinez I, Malaquias P, Hass V, Reis A, Campanha NH, et al. In vitro longevity of bonding properties of universal adhesives to dentin. Oper Dent. 2015 May-Jun;40(3):282-92. doi: 10.2341/14-055-L.

17. Valizadeh S, Moradi A, Mirazei M, Amiri H, Kharazifard MJ. Microshear bond strength of different adhesive systems to dentin. Front Dent. 2019 Jul-Aug;16(4):265-71. doi: 10.18502/fid.v16i4.2085.

18. Ikemura K, Endo T. Effect on adhesion of new polymerization initiator systems comprising 5-monosubstituted barbituric acids, aromatic sulphonate amides, and tert-butyl peroxymaleic acid in dental adhesive resin. J Appl Polym Sci. 1999 Apr;72(13):1655-68. doi: 10.1002/(SICI)1097-4628(19990624)72:13<1655::AID-APP2>3.0.C0;2-0.

19. Breschi L, Cadenaro M, Antoniolli F, Sauro S, Biasotto M, Prati C, et al. Polymerization kinetics of dental adhesives cured with LED: correlation between extent of conversion and permeability. Dent Mater. 2007 Sep;23(9):1066-72. doi: 10.1016/j.dental.2006.06.040.

20. Navarra CO, Cadenaro M, Armstrong SR, Jessop J, Antoniolli F, Sergo V, et al. Degree of conversion of filtek silorane adhesive system and clearfil SE bond within the hybrid and adhesive layer: an in situ Raman analysis. Dent Mater. 2009 Sep;25(9):1178-85. doi: 10.1016/j.dental.2009.05.009.

21. Flury S, Peutzfeldt A, Lussi A. Influence of increment thickness on microhardness and dentin bond strength of bulk fill resin composites. Dent Mater. 2014 Oct;30(10):1104-12. doi: 10.1016/j.dental.2014.07.001.

22. Peumans M, Wouters L, De Munck J, Van Meerbeek B, Van Landuyt K. Nine-year clinical performance of a HEMA-free one-step self-etch adhesive in noncarious cervical lesions. J Adhes Dent. 2018 20(3):195-203. doi: 10.3290/j.jad.a40630.

23. Van Meerbeek B, Yoshihara K, Yoshida Y, Mine A, De Munck J, Van Landuyt KL. State of the art of self-etch adhesives. Dent Mater. 2011 Jan;27(1):17-28. doi: 10.1016/j.dental.2010.10.023.

24. Scotti N, Cavalli G, Gagliani M, Breschi L. New adhesives and bonding techniques. Why and when? Int J Esthet Dent. 2017 12(4):524-35. 